\title{
RELAXATION PROCESSES
}

$\mathrm{A}^{\mathrm{N}}$ $\mathrm{N}$ informal discussion on "Relaxation Processes" was held under the auspices of the Faraday Society in the Department of Chemical Engineering, at the Imperial College of Science and Technology, London, on November 27. About eighty people from universities and industrial research centres both in Great Britain and abroad were present.

The chairman, Prof. A. R. Ubbelohde, welcomed the visitors and stressed the modern importance of relaxation techniques in the study of a wide variety of rate processes of interest to the physical chemist. The use of methods involving cyclic stresses could provide a valuable means of studying rate processes in systems only slightly perturbed from their equilibrium states, and made possible the correlation of rates with thermodynamic functions. This was of particular interest in the cases where the relaxation times could be associated with specific molecular mechanisms.

Prof. C. J. F. Böttcher (University of Leyden) presented a paper on the phenomenological study of dielectric relaxation. $\mathrm{He}$ considered a dielectric with polarization (strain) in the same direction as the applied field (stress), for the case in which there is a lag between stress and strain. If the stress is prescribed to rise and fall as a simple step-function with time, then the strain shows a simple retardation effect. If, on the other hand, the strain is prescribed to have a step form, then the stress required to effect this shows a relaxation effect. Since in practical dielectric measurements we work with a prescribed stress, it would seem logical to refer to the measured time-constant as the retardation time. He outlined the equations describing the variation of dielectric constant and of the loss factor with frequency. Although the retardation and relaxation times are not in general the same, they may be shown to have a simple mathematical connexion in this case and each can be related to a molecular relaxation time, so that the distinction between retardation and relaxation is in the nature of a formal rather than a physico-chemical one. The dependence on temperature of the molecular relaxation time can be understood by considering the relaxation mechanism as an Eyring type rate-process. Identification of the molecular process responsible for the macroscopic relaxation is facilitated by the experimental fact that a single relaxation curve is often obtained.

Illustrating the comparatively simple behaviour which could occur in complex molecules, Prof. Böttcher reported measurements on bromobenzene and cyclohexanol, each of which showed a single relaxation curve and mixtures of which showed only two relaxation times shifted towards each other, rather than a complex distribution of relaxation times.

Dr. Vera Daniel (Electrical Research Association, Leatherhead) described the results of measurements on a group of long-chain alcohols where anomalous dielectric constants (higher than those expected from the number of hydroxyl groups) are connected with the existence of polymeric forms of hydrogen bonds, so that the energy differences between the two forms of different polarization are small. Co-existence of these different forms is unlikely in a perfect crystal but likely at structural imperfections. Measurements were described on a variety of crystals grown from the melt or solution, containing different proportions of structural defects. These measurenents, together with those of other investigators, indicate that chains of hydrogen bonds become polarizable in relation to some kind of flaw. For a maximum dielectric constant fairly long chains are necessary, combined with a sufficiency of flaws. The flaws occupy space and a texture was suggested in the materials which would give suitable flaws to explain the dielectric properties and a number of other properties of the crystals.

Mr. W. Reddish (Imperial Chemical Industries, Plastics Division), in a paper on solid polymers, indicated that the way in which polymers soften as temperature increases is dependent on time and hence possess relaxation characteristics. Results of detailed measurements of harmonic dynamic mechanical moduli and losses show strong correlation with measurements of dielectric relaxation obtained at the same frequency, in the case of several important polar polymers. Measurements of the mechanical moduli over a wide range of temperature together with dielectric measurements over a wide range of frequency show that cases of softening by a single process are rare; often two or more processes are observed widely separated on the temperature scale, a polycarbonate for example showing processes $250^{\circ} \mathrm{C}$. apart. The number of processes possible seems to be related to the number of separate modes of rotational motion which can occur in the molecule, relatively free rotation of small groups occurring at low temperatures. These rotational motions may be inhibited in crystalline regions.

After comparison of dielectric and mechanical data for a range of compounds (especially when side-chains are varied systematically), it has sometimes been possible to suggest that particular relaxation processes are associated with motions of particular molecular groupings. Detailed models were discussed for polymethyl methacrylate and polymethyl chloracrylate. Another phenomenon of interest was that certain modes of motion observable mechanically but not electrically for non-polar polymers can be excited by adventitious dipolar impurities; for example, this function is attributed to carbonyl groups in polyethylene. Dielectric data for three processes for linear and branched slightly oxidized polyethylene were represented by means of solid models shown at the meeting.

In general discussion, Dr. C. W. Bunn (Imperial Chomical Industries, Plastics Division) raised a number of points connected with the hydrogenbonded structures suggested by Dr. Daniel. Dr. R. B. Beevers (Courtaulds, Maidenhead) pointed out that the high values of the dielectric constant obtained by Dr. Daniel for specimens prepared from the melt may possibly arise as a consequence of the formation of a meso-phase in the melting region and suggested that it would be worth while to determine if such a meso-phase existed.

Prof. G. Gee (University of Manchester) criticized methods of analysing the dependence of relaxation times on temperature by means of the equation :

$$
\log \tau=\log \tau_{0}+E / R T
$$

He pointed out, however, that a more precise analysis still gave remarkably large activation energies 
associated with the relaxation process in high polymers and suggested that this was because these were concerned with disturbances over large molecular areas of the material and not only with one bond.

Questions by Prof. Ubbelohde, Dr. Mansel Davies (University College, Aberystwyth) and Mr. B. Warburton (Distillers Co.) on matters arising from his paper were answered by Mr. Reddish. Dr. Greaverson (Shell) presented measurements on mechanical relaxation in polyethylene, Dr. G. Allen (University of Manchester) measurements on mechanical relaxation on rubber, and Dr. C. Brot (Paris) reported dielectric studies on liquid mono-alcohols of medium chain-length which showed three dispersion regions; these he interpreted in terms of molecular mechanisms.

In the second part of the meeting, Dr. J. H. Andrea (Imperial Chemical Industries, Research Department) presented results of ultrasonic relaxation measurements in liquids, ranging from low frequencies $(100 \mathrm{kc} . / \mathrm{s}$.$) where chemical equilibria$ involving rotational isomers may be perturbed by the sound wave and ultrasonic absorption observed, to the high frequencies $(100 \mathrm{Mc} / \mathrm{s}$.) where associationdissociation equilibria or slow redistribution of energy between translation and vibration may give rise to measurable ultrasonic absorption.

Dr. Andrea outlined ultrasonic measurements made by Dr. Lamb and his collaborators (Imperial College of Science and Technology, London) which gave values for the heats and entropies of activation in the passing from one rotational isomeric form to another, with particular reference to the substituted cyclohexanes. This gave a new method of measuring potential barriers hindering internal molecular rotation. He described the existence of an anomalous absorption region in toluene the origin of which is as yet unexplained.

Mr. J. Barlow (Imperial College of Science and Technology, London) described research in conjunction with Dr. Lamb, in which ultrasonic techniques were being used to determine the shear mechanical impedance of lubricating oils, as part of an investigation into the influence of the relaxation behaviour of molecules on lubrication. Measurements had been made for frequencies of the cyclic shearing stress in the region $10^{4}-10^{8} \mathrm{c} . / \mathrm{s}$. : the effective range could be extended by variation of the temperature and pressure of the oil under test. All the experimental results for a given oil could be reduced to a single curve for each component of the complex shear modulus. This information was then analysed in terms of a spectrum of relaxation times. Qualitative correlation could be made between the relaxation spectrum and the proportions of the various hydrocarbon types in the oil.

Dr. J. C. McCoubrey (Imperial College of Science and Technology, London) gave a résumé of work on ultrasonic relaxations in gases which could be used to give quantitative transition probabilities for the process in which a molecular vibration was de-excited from the first vibrational level to the ground-state by a collisional mechanism. Some theoretical explanation of these transition probabilities in terms of molecular parameters could be given, but this was inadequate to explain the dependence of ultrasonic relaxation times on temperature. A new technique involving the use of interferometry to study the changes in density across a shock front in gases in which ultrasonic relaxation had been observed could yield measurements of the translation-vibration transition probabilities at temperatures up to $3,000^{\circ} \mathrm{K}$., providing valuable tests for theories over a wide temperature-range.

In the general discussion, possible explanations of ultrasonic relaxation observed in toluene were sug. gested by Dr. J. A. Pople (National Physical Laboratory), Prof. H. C. Longuet-Higgins (Cambridge) and Dr. N. Sheppard (Cambridge). Some recent measurements on ultrasonies in gases were discussed by Mr. J. D. Lambert (Oxford), Dr. A. MacColl (University College, London) and Mr. T. L. Cottrell (Imperial Chemical Industries, Ltd.).

Finally, two new techniques giving information about relaxation times in energy transfer in gases were discussed. Dr. R. W. B. Stephens (Imperial College of Science and Technology, London) described an apparatus in which interrupted infra-red radiation is absorbed by a gas, and the vibrational energy taken up reappears as translational energy in the form of a pressure rise out of phase with the radiation input. The lag in phase depends on the relaxation time and this has been used to study relaxations in carbon monoxide and carbon dioxide.

Dr. A. G. Gaydon (Imperial College of Science and Technology, London) described an adaptation of the sodium-line reversal technique to the measurement of temperature changes in shock waves in gases showing relaxation. This procedure is probably capable of giving relaxation times at high temperatures more accurately than the interferometric study of density changes. J. C. MCCOUBREY

\section{PLANT MORPHOGENESIS}

A SYMPOSIUM on "Experimental Approaches to the Problems of Growth and Form in Plants", arranged by the Experimental Morphology Group of the Linnean Society, was held under the auspices of the Society in London during December 19-20. The symposium attracted a large and representative audience and was, in fact, an unusually interesting and successful occasion. It is probably true to say that this was one of the most varied and comprehensive treatments of plant morphogenesis that has been witnessed in Great Britain in recent years. (So considerable was the audience that the meetings had to be transferred to the rooms of the Geological Society.)
The papers presented at the symposium, some twenty in all, covered a wide range and explored the many aspects of morphogenesis by diverse experimental techniques. This wide spread of interest brought its own difficulties-the usual one on such occasions-that there was, unfortunately, all too little time for discussions.

It had been arranged that some of the longer contributions should be in the nature of general surveys. The opening paper, by Prof. C. W. Wardlaw, was of this kind, and dealt with observational, experimental and analytical methods which have contributed to our understanding of plant morphogenesis ; Prof. L. J. Audus discussed both in general terms 